\title{
Visualization of chromatin higher-order structures and dynamics in live cells
}

\author{
Tae Lim Park ${ }^{1, \#}$, YigJi Lee ${ }^{1, \#} \mathcal{E}$ Won-Ki Cho ${ }^{1,2, *}$ \\ ${ }^{1}$ Department of Biological Sciences, Korea Advanced Institute of Science and Technology (KAIST), Daejeon 34141, ${ }^{2} \mathrm{KI}$ for Health Science \\ and Technology (KIHST), Korea Advanced Institute of Science and Technology (KAIST), Daejeon 34141, Korea
}

Chromatin has highly organized structures in the nucleus, and these higher-order structures are proposed to regulate gene activities and cellular processes. Sequencing-based techniques, such as $\mathrm{Hi}-\mathrm{C}$, and fluorescent in situ hybridization (FISH) have revealed a spatial segregation of active and inactive compartments of chromatin, as well as the non-random positioning of chromosomes in the nucleus, respectively. However, regardless of their efficiency in capturing target genomic sites, these techniques are limited to fixed cells. Since chromatin has dynamic structures, live cell imaging techniques are highlighted for their ability to detect conformational changes in chromatin at a specific time point, or to track various arrangements of chromatin through long-term imaging. Given that the imaging approaches to study live cells are dramatically advanced, we recapitulate methods that are widely used to visualize the dynamics of higher-order chromatin structures. [BMB Reports 2021; 54(10): 489-496]

\section{INTRODUCTION}

Chromatin is hierarchically arranged in the cell nucleus (1). Accumulating evidence from current studies suggests that the organization of the three-dimensional (3D) genome is critical for regulating gene expressions and maintaining various cellular functions (2-8). To investigate large-scale chromatin organization, chromatin conformation capture (3C) methods have been widely used (9-11). Such sequencing-based techniques can efficiently identify contacts between chromatin domains and genomic loci by proximity ligation of cross-linked DNA fragments from fixed cells. Hi-C experiments recently revealed higher order chromatin organization that defines two types of segregated

*Corresponding author. Tel: +82-42-350-2647; Fax: +82-42-350-2610; E-mail: wonkicho@kaist.ac.kr

${ }^{\text {E}}$ These authors contributed equally to this work.

https://doi.org/10.5483/BMBRep.2021.54.10.098

Received 16 July 2021, Revised 9 August 2021 Accepted 29 August 2021

Keywords: Chromatin structure, Chromosome territories, Fluorescence imaging, Lamina-associated domains, Nucleolus associated domains chromatin interactions-compartment $\mathrm{A}$, and compartment $\mathrm{B}$ (Fig. 1) $(10,12)$. These two compartments have distinct characteristics. Compartment $\mathrm{A}$ is known to have open and accessible chromatin with higher transcription activity, while compartment $B$ is associated with compactly arranged, closed chromatin with lower gene density. In addition to genomic compartmentalization, the topologically associating domain (TAD) was identified through $\mathrm{Hi}-\mathrm{C}$ experiments, whose boundaries are associated with insulator binding protein CCCTC-binding factor (CTCF) and cohesion complex to form self-interacting chromatin regions at megabase scale (Fig. 1) (13).

Although sequencing-based techniques can identify long-range chromatin interactions and detect the spatial organization of chromosomes in the nucleus, these methods require millions of cells to calculate the averaged pattern of chromosome conformations. Since ensemble averaged data from the techniques underestimated cell-to-cell variability, the single-cell Hi-C method has been developed (14-18). However, regardless of the groundbreaking development of $\mathrm{Hi}-\mathrm{C}$ analysis, what still remains as challenging is that sequencing-based approaches cannot detect dynamic chromatin movements, as their protocols rely on fixed cells. Chromatins are known to have dynamic properties, of which their structural changes or positioning can affect numerous nuclear activities, including transcription, replication, and DNA repair. Thus, live-cell imaging has become essential for understanding spatiotemporal chromatin movements and related functions as clustered regularly interspaced short palindromic repeats (CRISPR)-based imaging systems and their applications combined with fluorescence super-resolution microscopy have been widely used to elucidate unknown nuclear mechanisms.

In this review, we discuss the development of microscopy techniques and strategies for visualizing the real-time dynamics of genomic organization. The major nuclear structures that we focus on are chromosome territories (CTs), lamina-associated domains (LADs), and nucleolar-associated domains (NADs), of which their structures are closely related to gene-expression regulation and cell-type specificity. 


\section{MAIN TEXT}

\section{Visualization of chromosome territories}

Genomic organization in the nucleus has been considered crucial for gene regulation and other nuclear processes. Followed by the advances in microscopic approaches, one key feature of nuclear architecture was revealed that chromosomes are not randomly positioned in the interphase nucleus, but instead occupy distinct nuclear spaces $(1,19-22)$. Such spatial segregation of individual chromosome is termed CT (Fig. 1). The most commonly used technique to study CTs is $\operatorname{FISH}(23,24)$.

To perform FISH, probes that consist of oligonucleotides to label a genomic region of interest are hybridized to the target chromosome, and enabled to visualize each CT (25). To further investigate the spatial arrangement of chromosomes, 3D FISH has been developed to generate $3 \mathrm{D}$ image reconstruction that is accomplished by confocal microscopy that could obtain serially sectioned nuclei, and stack all the images, to ultimately provide the 3D locations of chromosomes $(26,27)$. With 3D FISH technique, a critical pattern of genomic arrangement was discovered. Human (HSA) chromosome 19 with high gene density was found in the nuclear interior, while HSA 18 with low gene density was located at the nuclear periphery (28). A similar test was performed on HSA 12; gene-dense and gene-poor sections on the same chromosome were separately labeled

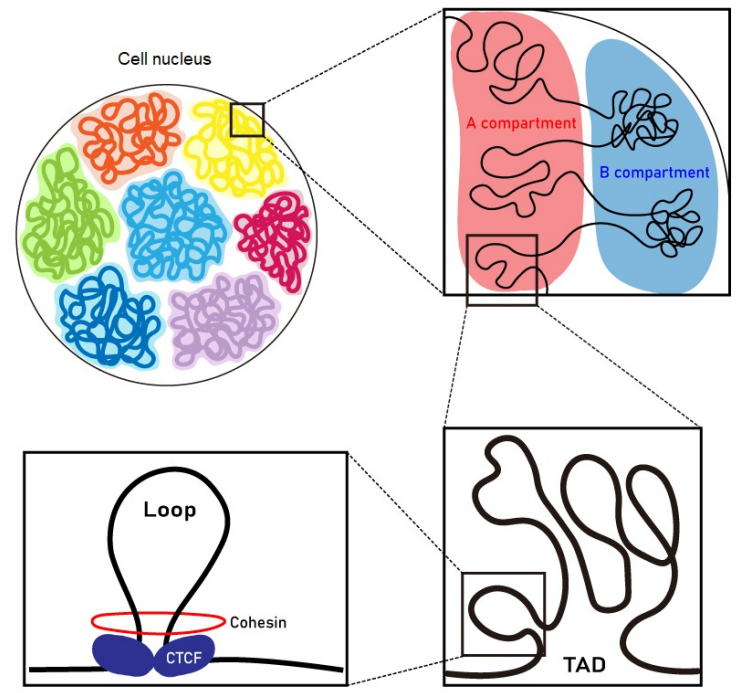

Fig. 1. Representations of higher-order chromatin organization. Hierarchical organization of chromatin is found in the cell nucleus. Each chromosome is positioned in spatially segregated regions called chromosome territories (CTs). Chromatin has two types of interactions, A compartment (red), and B compartment (blue). The A compartment is known have higher gene density, while the B compartment demonstrates lower gene density. Each compartment is composed of topologically associating domains (TADs), which are self-interacting chromatin regions. CCCTC-binding factor (CTCF) and cohesion complex bind to the TAD boundaries, and form chromatin looping structures. with two different probes, and the resulting 3D reconstruction image correlated with the previous finding. Regions with more genes tended to be positioned in the interior, while those with lower genes were found in the peripheral sites of the nucleus. These observations confirmed that gene density is closely related to the radial positions of chromosomes. Further studies also highlighted that among primates, the non-random positions of CTs are evolutionarily conserved, while they are significantly different in various cell types.

However, FISH-based techniques are limited to fixed cells, and involve DNA denaturation, which cannot provide information on the spatiotemporal changes of natural chromatin structures. To overcome the existing limitations, a number of approaches have been developed to achieve live-cell imaging, which allows the real-time dynamics of chromatin organization to be observed. The most well-known method applied for visualizing living cells is the clustered regularly CRISPR-based imaging system.

\section{CRISPR/dCas9 systems for genome labeling and dCas9} orthologs for multi-color imaging in live cells

The CRISPR technique was initially introduced for genome engineering, in combination with single-guide RNA (sgRNA), which contains specific sequences targeting a genomic region of interest, and CRISPR-associated protein 9 (Cas9), which has

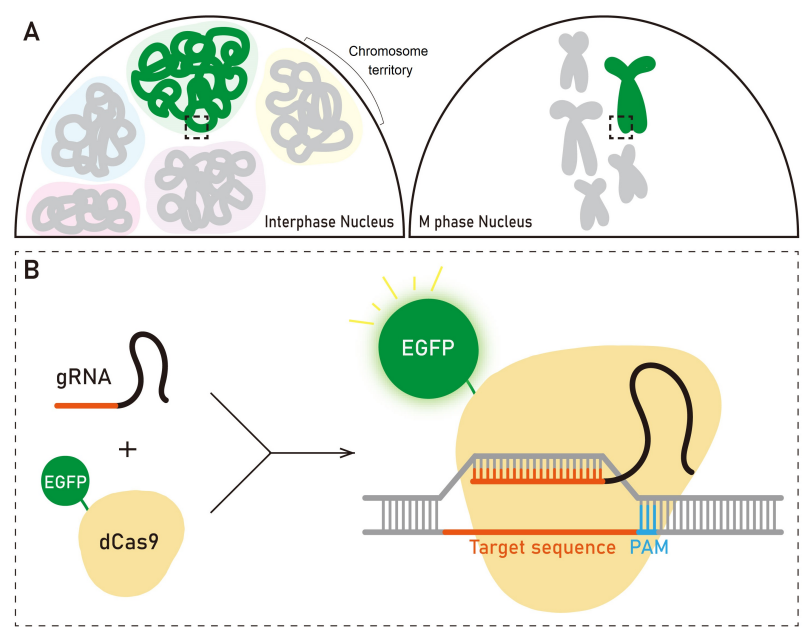

Fig. 2. Chromosome labeling with the CRISPR/dCas9 system in live cells. (A) Decondensed chromosomes are found in the interphase nucleus, and each of them occupies distinct nuclear spaces known as CTs. On the other hand, condensed chromosomes are found in the nucleus at mitotic phase. Decondensed and condensed chromosomes have different conformations, and these structures of the target chromosome (green) can be observed with the CRISPR/dCas9 labeling system. (B) The CRISPR/dCas9 system requires dCas9 fused with a fluorescent protein (in this case, EGFP is depicted) and gRNA. dCas9 with the fluorescent protein forms a complex with gRNA, and is directed to a target sequence with PAM. Then, this complex binds to the specific genomic site via gRNA-DNA hybridization, and fluorescent signals at this location can be tracked using various microscopic approaches. 
nuclease activity to cleave the targeted site, and induce DNA repair for genome editing (29-31). The mechanism that can recruit CRISPR/Cas9 complex to the specific genomic site via sgRNAs has made this technique suitable for genome imaging. To label the genome in live cells, Cas9 protein is modified to dead Cas9 (dCas9), which has deactivated nuclease function, and is fused with a fluorescent protein (32). Fluorescently labeled dCas9 complexed with CRISPR is recruited to the genomic region where sequences complementary to the specific sgRNA reside (Fig. 2B). The process required to select and generate appropriate sgRNAs is much simpler and easier than that for other methods, such as the transcription-activator-like effector nuclease (TALEN)-based technique, which involves specific protein productions for each targeting region. Thus, the CRISPR/ Cas9 system is extensively utilized for tracking the dynamics of genomic structures.

Genome labeling with the CRISPR/Cas9 technique can also be used to visualize multiple genomic regions (33). There are several orthogonal Cas9 variants that have different protospacer adjacent motif (PAM) sequences. PAM is a short DNA sequence downstream of genomic site targeted by sgRNA, and such diversity and specificity in PAM enable multi-locus labeling with Cas9 orthologs. The most commonly used dCas9 is from Streptococcus pyogenes (SpdCas9) with the PAM sequence 5'NGG-' ${ }^{\prime}$. Other known orthogonal Cas9 variants are from Staphylococcus aureus (SadCas), Neisseria meningitidis (NmdCas9), and Streptococcus thermophilus (St1dCas9), which recognize 5'-NNGRRT-3', 5'-NNNNGATT-3', and 5'-NNAGAAW-3', respectively. Recent studies tested whether these orthologs could be used in a combination to label interchromosomal and intrachromosomal loci in live cells, and their results confirmed that each ortholog distinctively labeled the targeted genomic region with high specificity achieved by the unique PAM sequences (34).

\section{Chromosome painting with CRISPR/Cas9 system}

Because the number of sgRNAs determines the range of labeled genomic sites and quality of fluorescent signal at the targeted spot, sgRNAs containing many repetitive sequences and low off-target bindings are generally selected for the CRISPR/Cas9 imaging system (32). However, sgRNA with repeated sequences can hardly target an entire chromosome. To overcome this challenge, one method has been recently proposed for whole chromosome painting with the CRISPR system by applying a large number of sgRNAs with non-repetitive sequences (35). In an attempt to label the entire chromosome 9, sgRNAs with (45-65)\% GC content were selected to satisfy adequate binding affinity, and were designed to not recognize places near transcription start sites, to avoid disruptions of transcription activity. A set of $30 \mathrm{sgRNAs}$ formed a cluster with $5 \mathrm{~Kb}$ spanning size, and a total of 15 clusters were chosen to be distributed throughout the entire chromosome 9. This approach successfully visualized chromosome $9 \mathrm{CT}$ in interphase, and at different stages of mitotic phase, from living HeLa cells (Fig.
2A). Some clusters were tested with FISH probes targeting the same sites where sgRNAs were recruited, to verify the accuracy of CRISPR painting. The regions labeled with CRISPR system and with FISH probes were detected as being co-localized, and this outcome confirmed the efficiency of the CRISPR-based method for visualizing the whole chromosome.

Although the outstanding capability of site-specific labeling has made the CRISPR/Cas9 imaging technique competent for tracking the live genomic structure, there is a challenging question that remains to be considered for further research: what is the unknown effect of the persistent occupancy of CRISPR/Cas9 complex on the genomic regions? In the case of the CRISPR chromosome painting method, one protein-coding gene, known as DNAJB5, was tested for its expression level when sgRNAs had binding sites on its exon (35). The result highlighted that the CRISPR/Cas9 complex did not affect its gene activity. For other cases, targeting repetitive sequences in introns of protein-coding genes is typically used for live genomic loci imaging, which is validated for its low perturbation of the gene expression level (35). However, the effect of CRISPR/ Cas9 complex on native genomic structures should be further studied. Hence, the most important aspect in CRISPR-mediated live genome labeling is selecting the appropriate sets of sgRNAs with low off-target effects, sufficient number of targeting sites for visible fluorescent signals, and minimal influence on the natural transcription level and chromatin structures.

\section{CARGO-dCas9 and CRISPR LiveFISH for enhanced visualization}

Despite its efficiency in multi-color labeling and live genome tracking, CRISPR/Cas9 mediated imaging often struggles to achieve better signal-to-noise ratio (SNR), which defines a measure comparing target signal level to background noise level. Based on the conventional approach, sgRNAs with repeated sequences are commonly used to obtain robust signal intensity, because delivering a single, highly repetitive sgRNA to target cells with transfection is simple. On the other hand, if non-repetitive sequences are selected, a large number of sgRNAs should be transferred to the target cells. To achieve high fluorescence signals, a strategy termed as chimeric array of gRNA oligonucleotides (CARGO) was developed to effectively and uniformly deliver sgRNAs with non-repetitive sequences (36). According to a widely used protocol for CRISPR/Cas9-based imaging, the target cell line stably expressing dCas9 with fluorescent protein is transduced by lentivirus encoding gRNA unit, which contains U6 promoter, RNA Polymerase III terminator, and guide RNA (gRNA) scaffold. However, CARGO assembly consists of a multiplexed gRNA array that is constructed by each gRNA unit cut with Bpil restriction enzyme to generate complementary sticky ends, and ligated with destination vector. The final product has multiple gRNA units cloned into a single vector. By tracking Fgf5 enhancer labeled with CARGO-dCas9EGFP (enhanced green fluorescent protein), this technique was confirmed as not interfering with transcription activity, while 
having high SNR with high specificity.

In addition to CARGO-dCas9 system, an alternative strategy to improve SNR has recently been proposed, and is known as CRISPR LiveFISH (37). Unlike the conventional CRISPR/Cas9based approach that uses fluorescent protein fused dCas9 for signal detection, gRNA tagged with a synthetic organic dye, cyanine 3 (Cy3), is used to track targeted regions. dCas9-EGFP and Cy3-gRNA targeting the same repetitive site in chromosome 3 were tested to compare their signal intensity and SNR, respectively. The results proved that both relative fluorescence and SNR were found to be much higher in the region labeled with Cy3-gRNA. Moreover, long-lasting expression of Cy3-gRNA was detected by forming more stable dCas9 complex. gRNAs are known to be easily degraded in the cellular environment. However, Cy3-gRNA presents higher stability when compared to the conventional gRNA used with fluorescently labeled dCas9, enabling long-term live cell imaging. Another advantage of CRISPR LiveFISH is that this method overcomes a nucleolar accumulation of dCas9, a common phenomenon in which dCas9 proteins not bound to gRNAs are frequently enriched in the nucleoli. Since CRISPR LiveFISH only detects target-bound dCas9-Cy3gRNA complex, it is applicable for spotting specific genomic sites without signals in the nucleoli.

\section{Mapping LADs and NADs with sequencing approaches}

Accumulated evidence from recent studies implies that the major structural components in the nucleus, nuclear membrane, and nucleolus could be considered as genome organizers, which affect gene expression regulatory processes $(38,39)$. The nuclear membrane is structured with double lipid bilayers composed of an outer nuclear membrane (ONM), and an inner nuclear membrane (INM). Below the INM, nuclear lamina is organized through a fibrillar meshwork of nuclear lamins, Atype and B-type lamins, which serve as a scaffold for the binding of various proteins, and chromatin domains as well. A-type lamins, which mainly include lamin $A$ and $C$, are encoded by $L M N A$, while B-type lamins are classified as lamin B1 and B2, coded by $L M N B 1$ and $L M N B 2$, respectively (40, 41). Another genome organizer, the nucleolus is the largest subcellular compartment in the nucleus, and is known as a site for ribosomal RNA (rRNA) synthesis and ribosome biogenesis. The nucleolus does not have a membrane, even though it is the largest sub-organelle inside the nucleus, while it consists of three layers forming a concentric arrangement: fibrillar center (FC), dense fibrillar component (DFC), and granular component (GC) (38). Numerous sequencing-based experimental approaches and studies with fluorescence imaging have revealed that the nuclear lamina and nucleolus represent the hubs for the chromatin organization, especially through interacting with heterochromatin domains.

Heterochromatin is spatially segregated into two main regions within the nucleus: nuclear periphery, and nucleolar periphery. The genomic regions that make molecular contacts with the nuclear lamina are termed lamina-associated domains (LADs)
(42-45), while the other genomic sites that frequently interact with the nucleolus are termed nucleolus-associated domains (NADs) $(46,47)$.

LADs are found on all chromosomes, covering around $40 \%$ of the entire genome. They typically range in length from (100 $\mathrm{kb}$ to $10 \mathrm{Mb}$ ), with a median size of $0.5 \mathrm{Mb}$. The human LADs are characterized as consisting of genes in transcriptionally silent state, or with low expression levels, and overlap with relatively gene-poor chromatin domains. LADs are especially enriched in B compartments with repressive histone modifications, such as di- and tri-methylated histone $\mathrm{H} 3$ lysine 9 (H3K9me2 and H3K9me3) $(43,48)$. A recent study with mouse embryonic stem cells has classified three types of chromatin regions in terms of LADs: constitutive LADs (cLADs), facultative LADs (fLADs), and constitutive interLADs (ciLADs). In detail, cLADs are defined as regions that remain in position during differentiation, while fLADs represent regions that interact with the nuclear lamina in a cell-type specific manner. ciLADs are sites that are outside of LADs in any cell type $(45,49)$. Interestingly, cLADs with the lowest gene density are highly conserved in their genomic positions, suggesting that cLADs play a crucial role in forming a "structural backbone" of chromatin organization in the nucleus $(50,51)$.

Similarly, NADs have low gene density, have a repressive chromatin signature, and contain a high density of AT-rich sequence elements. NADs have a comparable size range of (0.1-10) Mb and median sequence length (749 kb) to those of LADs $(46,47)$. There are two distinct classes of NADs: Type I NADs, and Type II NADs. Type I NADs commonly associate with both the nucleolar periphery and nuclear lamina, and exhibit features of constitutive heterochromatin. In contrast to Type I NADs, Type II NADs interact with nucleoli, but not with nuclear lamina (52). However, compared to LADs, NADs have been less thoroughly studied for their biology. Altogether, LADs and NADs share the general characteristics of heterochromatin.

How could these heterochromatin domains be identified? Genome-wide mapping studies of LADs and NADs were performed on multiple eukaryotic species, including Caenorhabditis elegans, Drosophila melanogaster, mouse, and human cells via several distinct methods, such as DNA adenine methyltransferase identification (DamID), chromatin immunoprecipitation sequencing (ChIP-seq), and NAD-seq $(42-45,48,53)$.

LADs were firstly identified by DamID, a proximity DNA labeling method. DamID works by fusing a bacterial adenine methyltransferase (Dam) to a protein of interest. To detect LADs, Dam is fused with lamin B1. Dam methylase recognizes GATC motifs in lamin B1-binding regions and methylates GATC sites, resulting in 6-methyl-adenine $\left({ }^{\mathrm{m} 6} \mathrm{~A}\right)$. Because ${ }^{\mathrm{m} 6} \mathrm{~A}$ modification is not normally detected in eukaryotes, DamID achieves specific labeling of lamina-interacting regions. Those regions containing ${ }^{\mathrm{m} 6} \mathrm{~A}$ are isolated by cutting with the methyl-specific restriction enzyme, Dpnl. These methylated fragments are selectively amplified and identified by DNA microarrays or high- 
throughput sequencing (DamID-seq) (43). DamID has emerged as a comprehensive technique to profile genome-wide proteinDNA interactions, and construct a high-resolution map for protein interaction sites in the genome. Beside the DamID method, LADs have also been mapped with ChIP-seq of A- or B-type lamins using lamin-specific antibodies (54-56).

NADs have been analyzed genome-wide through a combination of fluorescence comparative genome hybridization $(\mathrm{CGH})$ and high-throughput deep DNA sequencing with biochemically isolated nucleoli from nuclei, referred to as NAD-seq $(46,47)$. In addition to sedimentation, which is one of the nucleolar isolation methods, it has been reported that nucleoli from Arabidopsis thaliana leaves can be purified by introducing chimeric protein consisting of fluorescent protein (FP) fused to fibrillarin (FBL), a nucleolar protein. Then, those nuclei that contain FBL-FP are sorted by fluorescence-activated cell sorting (FACS) (57). Other techniques used for NADs studies include Split-Pool Recognition of Interactions by Tag Extension (SPRITE), a method that does not rely on proximity ligation. This innovative method allows genome-wide detection of higher-order interactions that occur simultaneously, or across long-range distances, within the nucleus (58). However, since H3K9me3-marked heterochromatins are resistant to sonication and the experimental processes to isolate nucleoli vary, sequencing based on purified nucleoli through sonication can be responsible for obtaining biased maps of NADs (59). These limitations led to the establishment of another novel method, called 'Nucleolar-DamID'. By applying the existing DamID, Dam is fused with the histone, H2B, where in this adaptation, a nucleolar localization signal (NoLS, RKK RKKK) is inserted at the C-terminus (60). Through these sequencing approaches, the genome-wide maps and heterochromatic characteristics of LADs and NADs have been defined.

\section{Visualization of LADs and NADs in live cells}

Although sequencing approaches have improved our understanding of LADs and NADs, those methods still have several limitations, including the lack of coordination of spatial and temporal information. Real-time visualization in living cells has been considered a powerful method, as it enables observation of the spatial arrangement and dynamics of heterochromatin domains in the three-dimensional space of the nucleus.

The dynamics of LADs has been tracked in live single-cells using the DamID-derived imaging method known as "Am6-tracer" (Fig. 3A). Dpnl contains a domain that recognizes and selectively binds to ${ }^{\mathrm{m} 6} \mathrm{~A}$. Genetically encoded ${ }^{\mathrm{m} 6} \mathrm{~A}$-tracer is fused with enhanced green fluorescent protein (eGFP) in a truncated dpn1 fragment that includes the binding domain, and has no cleavage activity. Since ${ }^{\mathrm{m} 6} \mathrm{~A}$ is a stable covalent modification, ${ }^{\mathrm{m} 6}$ A-tagged LADs can be continuously tracked once DNA makes contact with the nuclear lamina, even if the interaction no longer occurs at the moment of imaging. Therefore, this "molecular contact memory" strategy can provide a microscopic "history tracking" of nuclear lamina-interacting LADs (61). Many studies that utilized ${ }^{\mathrm{m} 6} \mathrm{~A}$-tracer or its modified system

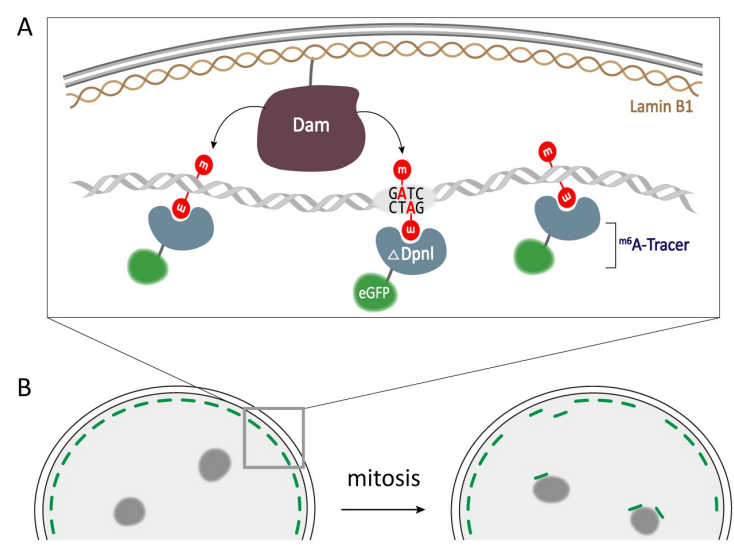

Fig. 3. Overview of DamlD-derived technology, ${ }^{\mathrm{m} 6} \mathrm{~A}$-Tracer for labeling LADs in live-single cells during mitosis. (A) Schematic of the ${ }^{\mathrm{m} 6} \mathrm{~A}$-Tracer system. The DamID-derived method, ${ }^{\mathrm{m} 6} \mathrm{~A}$-Tracer is applied to visualize Lamin-associated domains (LADs), which are large regions of heterochromatin that interact with the lamina at the nuclear periphery. This method for imaging LADs utilizes Dam (dark brown) fused to Lamin B1 (brown). Dam deposits methyl groups at the N6 positions of adenine residue $\left({ }^{\mathrm{m} 6} \mathrm{~A}\right.$, red) embedded in $5^{\prime}$-GATC-3' sites in DNA interacting with lamin B1. Catalytically inactivated Dpnl (blue) fused with eGFP (green) called ${ }^{\mathrm{m} 6} \mathrm{~A}$-Tracer specifically binds to ${ }^{\mathrm{m} 6} \mathrm{~A}$, resulting in LADs marked by ${ }^{\mathrm{m} 6} \mathrm{~A}$-Tracer. (B) $\mathrm{LAD}$ dynamics during mitosis. Studies using ${ }^{\mathrm{m} 6} \mathrm{~A}$-Tracer technique have demonstrated stochastic positioning of LADs. During interphase, the mobility of LADs is confined to a narrow zone; but after mitosis, they are redistributed throughout the nucleus of the daughter cells. A new subset of LADs interacts with the nuclear lamina, and nucleoplasmic LADs can be in contact with nucleoli (gray).

have demonstrated that during interphase, LADs have constrained mobility, while after mitosis, have appeared to be intrinsically stochastic (Fig. 3B). These observations suggest that LAD localization is generally not inherited. Interestingly, after mitosis, some of the nulceoplasmic LADs become closely associated with nucleoli $(61,62)$. These results are consistent with the genome-wide mapping studies in which NADs sequences partially overlap with LADs $(46,47)$.

Unlike the nuclear lamina, the nucleolus is a subnuclear membrane-less organelle (63), which makes it challenging to apply the DamID-derived tool to track NADs in live cells. This implicates that there seems to be no possible candidate molecules as scaffold to bind in and around the nucleoli, while there are the stable lamins under the nuclear envelope for mapping LADs. However, Nucleolar-DamID using Dam-fused and NoLS inserted histone, which can bind DNA sequences near nucleoli without motif specificity, has recently been proposed (60), thereby applying it may allow insight to be gained into the dynamics of NADs, as has been done for LADs. In addition to Nucleolar-DamID, other creative technologies for visualizing NADs need to be established.

Live cell imaging is an attractive analytical methodology for understanding cellular structures and their dynamics. It can 
provide intuitive and dynamic information over time, which are possibly lost in snapshots from fixed cells. Heterochromatin is critical to the $3 \mathrm{D}$ organization of the interphase genome, thereby imaging it in real-time will provide answers to the fundamental question of which mechanisms are involved in the spatial genome organization.

\section{DISCUSSION}

Recently, higher-order structures of chromatin have become more appreciated for their roles in gene regulation and cellular processes. Unlike sequencing-based methods or FISH that rely on fixed cells, microscopic approaches have been developed, and have opened an era for studying chromatin dynamics in live cells. With an attempt to visualize chromatin organization in living cells, there are many methods proposed for fluorescently labeling specific genomic regions, or whole chromosomes. One of the most well-known methods is the CRISPR/Cas9 system, and its modified techniques, such as CARGO-dCas9 and CRISPR LiveFISH, have recently been introduced to obtain enhanced results from live cell imaging. Moreover, there is another way to label genomic sites in live cells, called the DamID-derived technique. This system uses Dam protein fused with the protein of interest to methylate adenines, and generates ${ }^{\mathrm{m} 6} \mathrm{~A}$ modifications at specific regions of the genome. These modified DNA regions are recognized by catalytically inactivated Dpnl enzyme fused with fluorescent protein, which allows stable tracking of genomic structures, such as LADs and NADs.

\section{ACKNOWLEDGEMENTS}

This work was supported by the Suh Kyungbae Foundation and National Research Foundation of Korea (NRF) grants (2020 R1C1C1014599, 2020R1A4A3079755, 2019M3A9H1103711, and 2019R1A6A1A10073887 to W.-K. Cho).

\section{CONFLICTS OF INTEREST}

The authors have no conflicting interests.

\section{REFERENCES}

1. Cremer T and Cremer C (2001) Chromosome territories, nuclear architecture and gene regulation in mammalian cells. Nat Rev Genet 2, 292-301

2. Francastel C, Schubeler D, Martin DI and Groudine M (2000) Nuclear compartmentalization and gene activity. Nat Rev Mol Cell Biol 1, 137-143

3. Strahl BD and Allis CD (2000) The language of covalent histone modifications. Nature 403, 41-45

4. Dundr M and Misteli T (2001) Functional architecture in the cell nucleus. Biochem J 356, 297-310

5. Levine M, Cattoglio C and Tjian R (2014) Looping back to leap forward: transcription enters a new era. Cell 157,
13-25

6. Dekker J and Mirny L (2016) The 3d genome as moderator of chromosomal communication. Cell 164, 1110-1121

7. Bickmore WA and van Steensel B (2013) Genome architecture: Domain organization of interphase chromosomes. Cell 152, 1270-1284

8. Cremer T and Cremer M (2010) Chromosome territories. Cold Spring Harb Perspect Biol 2, a003889

9. Dekker J, Rippe K, Dekker M and Kleckner N (2002) Capturing chromosome conformation. Science 295, 1306-1311

10. Lieberman-Aiden $\mathrm{E}$, van Berkum NL, Williams $\mathrm{L}$ et al (2009) Comprehensive mapping of long-range interactions reveals folding principles of the human genome. Science 326, 289-293

11. Cullen KE, Kladde MP and Seyfred MA (1993) Interaction between transcription regulatory regions of prolactin chromatin. Science 261, 203-206

12. Rao SSP, Huntley MH, Durand NC et al (2014) A 3d map of the human genome at kilobase resolution reveals principles of chromatin looping. Cell 159, 1665-1680

13. Szabo Q, Bantignies F and Cavalli G (2019) Principles of genome folding into topologically associating domains. Sci Adv 5, eaaw 1668

14. Nagano T, Lubling Y, Vaarnai C et al (2017) Cell-cycle dynamics of chromosomal organization at single-cell resolution. Nature 547, 61-67

15. Flyamer IM, Gassler J, Imakaev M et al (2017) Singlenucleus hi-c reveals unique chromatin reorganization at oocyte-tozygote transition. Nature 544, 110-114

16. Stevens TJ, Lando D, Basu S et al (2017) 3d structures of individual mammalian genomes studied by single-cell hi-c. Nature 544, 59-64

17. Ramani V, Deng X, Qiu R et al (2017) Massively multiplex single-cell hi-c. Nat Methods 14, 263-266

18. Tan L, Xing D, Chang CH, Li H and Xie XS (2018) Threedimensional genome structures of single diploid human cells. Science 361, 924-928

19. Chevret E, Volpi EV and Sheer D (2000) Mini review: Form and function in the human interphase chromosome. Cytogenet Cell Genet 90, 13-21

20. Cremer T, Kreth G, Koester $\mathrm{H}$ et al (2000) Chromosome territories, interchromatin domain compartment, and nuclear matrix: An integrated view of the functional nuclear architecture. Crit Rev Eukaryot Gene Expr 10, 179-212

21. Lamond Al and Earnshaw WC (1998) Structure and function in the nucleus. Science 280, 547-553

22. Leitch AR (2000) Higher levels of organization in the interphase nucleus of cycling and differentiated cells. Microbiol Mol Biol Rev 64, 138-152

23. Langer-Safer PR, Levine M and Ward DC (1982) Immunological method for mapping genes on drosophila polytene chromosomes. Proc Natl Acad Sci U S A 79, 4381-4385

24. Lichter P, Tang CJ, Call K et al (1990) High-resolution mapping of human chromosome 11 by in situ hybridization with cosmid clones. Science 247, 64-69

25. Bolzer A, Craig JM, Cremer T and Speicher MR (1999) A complete set of repeat-depleted, pcr-amplifiable, human chromosome-specific painting probes. Cytogenet Cell Genet 84, 233-240

26. Croft JA, Bridger JM, Boyle S, Perry P, Teague P and Bickmore 
WA (1999) Differences in the localization and morphology of chromosomes in the human nucleus. J Cell Biol 145, 1119-1131

27. Habermann FA, Cremer M, Walter J et al (2001) Arrangements of macro- and microchromosomes in chicken cells. Chromosome Res 9, 569-584

28. Tanabe H, Muller S, Neusser M et al (2002) Evolutionary conservation of chromosome territory arrangements in cell nuclei from higher primates. Proc Natl Acad Sci U S A 99, 4424-4429

29. Jinek M, Chylinski K, Fonfara I, Hauer M, Doudna JA and Charpentier E (2012) A programmable dual-rna-guided DNA endonuclease in adaptive bacterial immunity. Science 337, 816-821

30. Cong L, Ran FA, Cox D et al (2013) Multiplex genome engineering using crispr/cas systems. Science 339, 819-823

31. Mali P, Yang L, Esvelt KM et al (2013) Rna-guided human genome engineering via cas9. Science 339, 823-826

32. Chen B, Gilbert LA, Cimini BA et al (2013) Dynamic imaging of genomic loci in living human cells by an optimized crispr/cas system. Cell 155, 1479-1491

33. Ma H, Tu LC, Naseri A et al (2016) Multiplexed labeling of genomic loci with dcas9 and engineered sgrnas using crisprainbow. Nat Biotechnol 34, 528-530

34. Ma H, Naseri A, Reyes-Gutierrez P, Wolfe SA, Zhang S and Pederson T (2015) Multicolor crispr labeling of chromosomal loci in human cells. Proc Natl Acad Sci U S A 112, 3002-3007

35. Zhou $Y$, Wang $P$, Tian $F$ et al (2017) Painting a specific chromosome with crispr/cas9 for live-cell imaging. Cell Res 27, 298-301

36. Gu B, Swigut T, Spencley A et al (2018) Transcriptioncoupled changes in nuclear mobility of mammalian cisregulatory elements. Science 359, 1050-1055

37. Wang H, Nakamura M, Abbott TR et al (2019) Crispr-mediated live imaging of genome editing and transcription. Science 365, 1301-1305

38. Bersaglieri C and Santoro R (2019) Genome organization in and around the nucleolus. Cells 8, 579

39. Briand $N$ and Collas $P$ (2020) Lamina-associated domains: Peripheral matters and internal affairs. Genome Biol 21, 85

40. Prokocimer M, Davidovich M, Nissim-Rafinia M et al (2009) Nuclear lamins: key regulators of nuclear structure and activities. J Cell Mol Med 13, 1059-1085

41. Shevelyov YY and Nurminsky DI (2012) The nuclear lamina as a gene-silencing hub. Curr Issues Mol Biol 14, 27-38

42. Pickersgill $H$, Kalverda B, de Wit E, Talhout W, Fornerod M and van Steensel B (2006) Characterization of the drosophila melanogaster genome at the nuclear lamina. Nat Genet 38, 1005-1014

43. Guelen L, Pagie L, Brasset E et al (2008) Domain organization of human chromosomes revealed by mapping of nuclear lamina interactions. Nature 453, 948-951

44. Ikegami K, Egelhofer TA, Strome S and Lieb JD (2010) Caenorhabditis elegans chromosome arms are anchored to the nuclear membrane via discontinuous association with lem-2. Genome Biol 11, 1-20

45. Peric-Hupkes D, Meuleman W, Pagie L et al (2010) Molecular maps of the reorganization of genome-nuclear lamina interactions during differentiation. Mol Cell 38, 603-613

46. Nemeth A, Conesa A, Santoyo-Lopez J et al (2010) Initial genomics of the human nucleolus. PLoS Genet 6, e1000889

47. van Koningsbruggen $S$, Gierlinski $M$, Schofield $P$ et al (2010) High-resolution whole-genome sequencing reveals that specific chromatin domains from most human chromosomes associate with nucleoli. Mol Biol Cell 21, 37353748

48. Kind J, Pagie L, de Vries SS et al (2015) Genome-wide maps of nuclear lamina interactions in single human cells. Cell 163, 134-147

49. Meuleman W, Peric-Hupkes D, Kind J et al (2013) Constitutive nuclear lamina-genome interactions are highly conserved and associated with a/t-rich sequence. Genome Res 23, 270-280

50. van Steensel B and Belmont AS (2017) Lamina-associated domains: Links with chromosome architecture, heterochromatin, and gene repression. Cell 169, 780-791

51. Bizhanova A and Kaufman PD (2021) Close to the edge: Heterochromatin at the nucleolar and nuclear peripheries. Biochim Biophys Acta Gene Regul Mech 1864, 194666

52. Vertii A, Ou J, Yu J et al (2019) Two contrasting classes of nucleolus-associated domains in mouse fibroblast heterochromatin. Genome Res 29, 1235-1249

53. Borsos M, Perricone SM, Schauer T et al (2019) Genomelamina interactions are established de novo in the early mouse embryo. Nature 569, 729-733

54. Shah PP, Donahue G, Otte GL et al (2013) Lamin b1 depletion in senescent cells triggers large-scale changes in gene expression and the chromatin landscape. Genes Dev 27, 1787-1799

55. Lund EG, Duband-Goulet I, Oldenburg A, Buendia B and Collas P (2015) Distinct features of lamin a-interacting chromatin domains mapped by chip-sequencing from sonicated or micrococcal nuclease-digested chromatin. Nucleus 6, 30-39

56. Gesson K, Rescheneder P, Skoruppa MP, von Haeseler A, Dechat T and Foisner R (2016) A-type lamins bind both hetero- and euchromatin, the latter being regulated by lamina-associated polypeptide 2 alpha. Genome Res 26, 462-473

57. Pontvianne F, Carpentier MC, Durut N et al (2016) Identification of nucleolus-associated chromatin domains reveals a role for the nucleolus in $3 \mathrm{~d}$ organization of the a. Thaliana genome. Cell Rep 16, 1574-1587

58. Quinodoz SA, Ollikainen N, Tabak B et al (2018) Higherorder inter-chromosomal hubs shape $3 \mathrm{~d}$ genome organization in the nucleus. Cell 174, 744-757 e24

59. Becker JS, McCarthy RL, Sidoli S et al (2017) Genomic and proteomic resolution of heterochromatin and its restriction of alternate fate genes. Mol Cell 68, 1023-1037. e15

60. Bersaglieri C, Kresoja-Rakic J, Gupta S, Bär D, Kuzyakiv R and Santoro R (2020) Genome-wide maps of nucleolus interactions reveal distinct layers of repressive chromatin domains. bioRxiv, 2020.11.17.386797

61. Kind J, Pagie L, Ortabozkoyun H et al (2013) Single-cell dynamics of genome-nuclear lamina interactions. Cell 153, 178-192

62. Kind J and van Steensel B (2014) Stochastic genome-nuclear 
lamina interactions: Modulating roles of lamin a and baf. Nucleus 5, 124-130

63. Birch JL and Zomerdijk JC (2008) Structure and function of ribosomal rna gene chromatin. Biochem Soc Trans 36, 619-624 